\title{
New information on photon fragmentation functions
}

\author{
Michael Klasen $^{\mathrm{a}}$, Florian König ${ }^{\mathrm{b}}$ \\ Institut für Theoretische Physik, Westfälische Wilhelms-Universität Münster, Wilhelm-Klemm-Straße 9, 48149 Münster, Germany
}

Received: 23 May 2014 / Accepted: 25 July 2014 / Published online: 23 August 2014

(C) The Author(s) 2014. This article is published with open access at Springerlink.com

\begin{abstract}
Thermal photons radiated in heavy-ion collisions represent an important signal for a recently discovered new state of matter, the deconfined quark-gluon plasma. However, a clean identification of this signal requires precise knowledge of the prompt photons produced simultaneously in hard collisions of quarks and gluons, mostly through their fragmentation. In this paper, we demonstrate that PHENIX data on photons produced in proton-proton collisions with low transverse momenta allow one to extract new information on this fragmentation process. While existing data do not yet convincingly favor one parameterization (BFG II) over the two other frequently used photon fragmentation functions (BFG I and GRV NLO), the data sets recorded by PHENIX and STAR at BNL RHIC in 2013 with tenfold higher statistics should allow for such an analysis.
\end{abstract}

\section{Introduction}

In the early Universe, at temperatures above a critical temperature of about $T_{\text {crit. }} \simeq 10^{12} \mathrm{~K}$ or $170 \mathrm{MeV}$, quarks and gluons are believed to have existed in a new, deconfined state of matter, before they were bound by strong interactions into protons and nuclei. Relativistic heavy-ion colliders such as BNL RHIC and CERN LHC allow one today to re-create this state, the so-called quark-gluon plasma (QGP), on earth, albeit only for very short times of about $10^{-23} \mathrm{~s}$. An important signal for the presence of a QGP and a good probe of its properties is the radiation of thermal photons with low transverse momenta (typically $\leq 4 \mathrm{GeV}$ ) from the deconfined partons before thermalization, in the thermal bath, during expansion and cooling of the QGP, and finally from the thermal hadron gas $[1,2]$.

The interpretation of inclusive photon measurements is complicated by the fact that photons are also produced in

\footnotetext{
a e-mail: michael.klasen@uni-muenster.de

be-mail: f.koenig@uni-muenster.de
}

hadron (mostly neutral pion) decays, which must be reliably subtracted from the experimental data, as well as in hard scatterings of the quarks and gluons in the colliding ions. At high transverse momenta, photons are mostly produced directly, whereas in the interesting low-transverse-momentum range they stem predominantly from quark and gluon fragmentation [3].

The probability for quark and gluon fragmentation into photons can unfortunately not be computed in perturbative QCD, but it must be parameterized with photon fragmentation functions (FFs) $D_{\gamma / q, g}\left(z, Q^{2}\right)$. Their dependence on the longitudinal momentum fraction $z$ transferred from the parton to the photon is unknown and therefore modeled at a starting scale $Q_{0}$. It is then evolved using QCD renormalization group equations to higher scales $Q$, where experimental data are available and can be used to constrain the theoretical ansatz.

Traditionally, these data have been taken from $e^{+} e^{-}$colliders in order to avoid theoretical uncertainties from the initial state and, in the absence of usable data on prompt photons, from the production of vector mesons [4,5], assuming that they dominate the hadronic fluctuations into the photon [6,7]. Today, however, the parton density functions (PDFs) in the proton are known with much better precision than the photon FFs [8], and a wealth of new data on prompt photon production has been taken in hadronic collisions [9]. In particular, the PHENIX collaboration at BNL RHIC have analyzed $4 \mathrm{pb}^{-1}$ of $2006 \mathrm{pp}$ collision data at $\sqrt{s}=200 \mathrm{GeV}$ for the production of nearly real photons with transverse momenta in the range $1 \mathrm{GeV}<p_{T}<5 \mathrm{GeV}$ using a single-electron trigger, which greatly reduced the background from light meson decays [10]. These data are complemented by and overlap with real-photon data in the range $p_{T}>4 \mathrm{GeV}$.

More recently, measurements of prompt photon production in hadron collisions have been performed at the Tevatron $[11,12]$ and the LHC $[13,14]$ (see also the references therein), albeit mostly at high $p_{T}$ and/orwith photon isolation, which 
reduces the sensitivity of these data to the photon fragmentation contribution.

In this paper, we demonstrate that prompt photon data from BNL RHIC allow one in principle to extract new information on the photon FFs. By separating the data into a control region of large transverse momenta (above $10 \mathrm{GeV}$ ) and a signal region (below $5 \mathrm{GeV}$ ) dominated by directly produced and fragmentation photons, respectively, we first establish the reliability of the FF-independent parts of our perturbative QCD calculation in the control region, before we perform chi-square tests of the three available modern FFs (BFG I, BFG II [6] and GRV NLO [7]) in the signal region.

\section{Photon fragmentation functions}

When a photon is radiated from a massless final-state quark, it exhibits a collinear singularity that must be absorbed into a non-perturbative FF $D_{\gamma / q}\left(z, Q^{2}\right)$. At next-to-leading order (NLO) of perturbative QCD, also gluons fragment into photons through intermediate quarks, which gives rise to the corresponding FF $D_{\gamma / g}\left(z, Q^{2}\right)$. The evolution of these FFs with the scale $Q$ is described by renormalization group equations [15],

$$
\begin{aligned}
\frac{\mathrm{d} D_{\gamma / q}\left(Q^{2}\right)}{\mathrm{d} \ln Q^{2}}= & \frac{\alpha}{2 \pi} P_{\gamma \leftarrow q} \otimes D_{\gamma / \gamma}\left(Q^{2}\right)+\frac{\alpha_{s}\left(Q^{2}\right)}{2 \pi} \\
& \times\left[P_{q \leftarrow q} \otimes D_{\gamma / q}\left(Q^{2}\right)+P_{g \leftarrow q} \otimes D_{\gamma / g}\left(Q^{2}\right)\right],
\end{aligned}
$$

$$
\begin{aligned}
\frac{\mathrm{d} D_{\gamma / g}\left(Q^{2}\right)}{\mathrm{d} \ln Q^{2}}= & \frac{\alpha}{2 \pi} P_{\gamma \leftarrow g} \otimes D_{\gamma / \gamma}\left(Q^{2}\right)+\frac{\alpha_{s}\left(Q^{2}\right)}{2 \pi} \\
& \times\left[P_{q \leftarrow g} \otimes D_{\gamma / q}\left(Q^{2}\right)+P_{g \leftarrow g} \otimes D_{\gamma / g}\left(Q^{2}\right)\right],
\end{aligned}
$$

$$
\begin{aligned}
\frac{\mathrm{d} D_{\gamma / \gamma}\left(Q^{2}\right)}{\mathrm{d} \ln Q^{2}}= & \frac{\alpha}{2 \pi} P_{\gamma \leftarrow \gamma} \otimes D_{\gamma / \gamma}\left(Q^{2}\right)+\frac{\alpha}{2 \pi} \\
& \times\left[P_{q \leftarrow \gamma} \otimes D_{\gamma / q}\left(Q^{2}\right)+P_{g \leftarrow \gamma} \otimes D_{\gamma / g}\left(Q^{2}\right)\right],
\end{aligned}
$$

which are coupled through the perturbatively calculable timelike Altarelli-Parisi splitting functions $P_{j \leftarrow i}$ [16]. Note that, contrary to the evolution equations of partons in hadrons, those of the photon also contain inhomogeneous terms related to its pointlike contribution.

In leading order ( $\mathrm{LO}$ ) of the electromagnetic coupling constant $\alpha$, the third evolution equation, Eq. (3), can be directly integrated with the result $D_{\gamma / \gamma}\left(z, Q^{2}\right)=\delta(1-z)$. Furthermore, in LO of the strong coupling constant $\alpha_{s}$, only the evolution equation of the quark-photon FF

$\frac{\mathrm{d} D_{\gamma / q}\left(z, Q^{2}\right)}{\mathrm{d} \ln Q^{2}}=\frac{\alpha}{2 \pi} P_{\gamma \leftarrow q}(z)$ survives, which can also be integrated with the result

$D_{\gamma / q}\left(z, Q^{2}\right)=\frac{\alpha}{2 \pi} P_{\gamma \leftarrow q}(z) \ln \frac{Q^{2}}{Q_{0}^{2}}+D_{\gamma / q}\left(z, Q_{0}^{2}\right)$.

The first term in Eq. (5) is the perturbatively calculable pointlike solution, while the second term is a hadronic boundary condition, which has to be fitted to experimental data.

In the modified Minimal Subtraction ( $\overline{\mathrm{MS}})$ scheme [17], the inclusive NLO cross section for $e^{+} e^{-} \rightarrow \gamma X$ is [18]

$$
\begin{aligned}
\frac{1}{\sigma_{0}} \frac{\mathrm{d} \sigma\left(Q^{2}\right)}{\mathrm{d} z}= & \sum_{q} 2 e_{q}^{2}\left\{D_{\gamma / q}\left(Q^{2}\right)+\frac{\alpha}{2 \pi} e_{q}^{2} C_{\gamma}+\frac{\alpha_{s}\left(Q^{2}\right)}{2 \pi}\right. \\
& \left.\times\left[C_{q} \otimes D_{\gamma / q}\left(Q^{2}\right)+C_{g} \otimes D_{\gamma / g}\left(Q^{2}\right)\right]\right\},
\end{aligned}
$$

where $\sigma_{0}=4 \pi \alpha^{2} N_{C} /\left(3 Q^{2}\right)$ is $N_{C}=3$ times the cross section for $e^{+} e^{-} \rightarrow \mu^{+} \mu^{-}, e_{q}$ is the fractional quark charge, the factor of two comes from $D_{\gamma / q}\left(Q^{2}\right)=D_{\gamma / \bar{q}}\left(Q^{2}\right)$, and $C_{q, g}$ stand for the time-like Wilson coefficients of transverse and longitudinal partonic cross sections. In the $\mathrm{DIS}_{\gamma}$ factorization scheme, the singular transverse photonic Wilson coefficient $C_{\gamma}^{T} \propto \ln \left[z^{2}(1-z)\right]$ can be absorbed into the quark FF, thereby increasing the perturbative stability [7].

The hadronic input in Eq. (5), and similarly for the gluon, can unfortunately not be determined from inclusive photon production in $e^{+} e^{-}$annihilation, since the experimental data are very limited and furthermore dominated by the pointlike quark-photon FF [19,20]. Therefore, all current parameterizations assume vector-meson dominance (VMD) of hadronic fluctuations into the photon to model the photon fragmentation at low scales. The most relevant input parameters are summarized in Table 1. In particular, BFG [6] work in the $\overline{\mathrm{MS}}$ scheme and choose a higher scale $Q_{0}$ and slightly larger QCD scale parameter $\Lambda$ for $N_{f}=4$ flavors than GRV [7], who use the DIS $\gamma$ scheme. Our perturbative calculation is then of course adjusted accordingly [9]. Heavy quarks of mass $m_{h}$ are included above their production thresholds with boundary conditions $D_{\gamma / h}\left(z, m_{h}^{2}\right)=D_{\gamma / \bar{h}}\left(z, m_{h}^{2}\right)=0$. As can be seen from Fig. 1, these assumptions lead to good agreement on the (mostly pointlike) quark FFs, but the gluon FFs differ widely (by up to an order of magnitude), even among BFG I and BFG II. The factorization scale $Q=\mu_{D}=2 \mathrm{GeV}$ has been chosen here in accordance with the typical transverse momenta to be analyzed below.

\section{Subprocess contributions}

In proton-proton collisions, photons are not only produced by fragmentation of the colliding quarks and gluons, but also 
Table 1 Current parameterizations of the photon FFs. $\rho, \omega$ and $\phi$ contributions can be added coherently or incoherently in vector-meson dominance (VMD) models. $N_{g}$ is the normalization of the gluon FF at the starting scale

\begin{tabular}{lllllll}
\hline Group & Set & Year & $Q_{0}^{2}\left(\mathrm{GeV}^{2}\right)$ & Factor. scheme & VMD model & $\Lambda_{\overline{\mathrm{MS}}}^{N_{f}=4}(\mathrm{MeV})$ \\
\hline BFG & I & 1998 & 2 & $\overline{\mathrm{MS}}$ & Coh., $N_{g}$ free & 230 \\
BFG & II & 1998 & 2 & $\overline{\mathrm{MS}}$ & Coh., $N_{g}$ fixed & 230 \\
GRV & NLO & 1993 & 0.3 & DIS & Incoherent & 200 \\
\hline
\end{tabular}

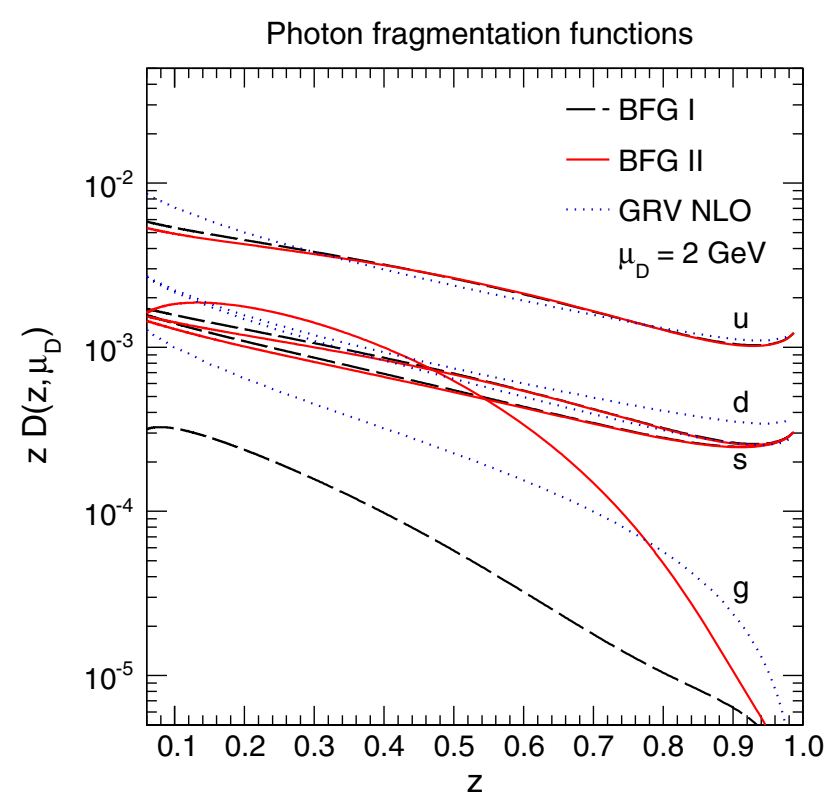

Fig. 1 Quark (up, down, and strange) and gluon ( $g$ ) FFs into photons at the scale $Q=\mu_{D}=2 \mathrm{GeV}$ as parameterized by the BFG [6] and GRV collaborations [7]

directly in processes like quark-antiquark fusion, $q \bar{q} \rightarrow \gamma g$, and QCD Compton scattering, $q g \rightarrow \gamma q$. Since we want to separate the PHENIX data set into a signal and a control region, dominated by fragmentation and direct production, respectively, we must first establish the corresponding $p_{T}$ regions. To this end, we compute the fractional subprocess contributions in NLO QCD using the program JETPHOX [9], assuming a fixed set of parton densities given by the CT10 parameterization [8], which are well constrained in the region of $x_{T}=2 p_{T} / \sqrt{s}=0.01-0.1$ relevant here, and identifying the renormalization scale $\mu_{R}$, the proton factorization scale $\mu_{F}$ and the photon fragmentation scale $\mu_{D}$ with the central hard scale of the process, the photon transverse momentum $p_{T}$. Figure 2 then shows that fragmentation processes dominate for $p_{T} \leq 5 \mathrm{GeV}$ in $p p$ collisions at $\sqrt{s}=200 \mathrm{GeV}$, while for $p_{T}>10 \mathrm{GeV}$ direct processes account for $60-75 \%$ of the total cross section, depending on $\mu_{D}$. If one wants to fix the fragmentation-independent parts of the NLO QCD calculation [9], it is therefore preferable to choose $\mu_{D}=0.5 p_{T}$ in order to minimize the fragmentation contribution.

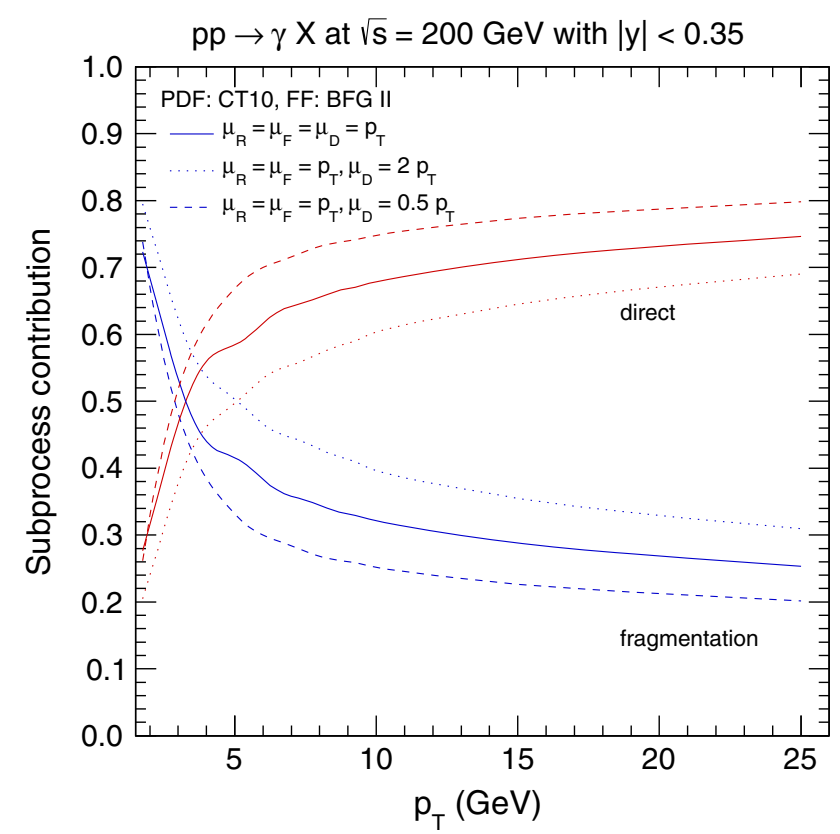

Fig. 2 Fractional contributions of direct and fragmentation processes to inclusive photon production at BNL RHIC as a function of $p_{T}$ for three different choices of the photon fragmentation scale $\mu_{D}$

\section{Comparison with PHENIX data}

Having fixed our signal and control regions as described above, we next allow all three scales to vary independently among the choices $(0.5 ; 1 ; 2) p_{T}$ in the control region $\left(p_{T}>\right.$ $10 \mathrm{GeV}$ ) and fit them to the PHENIX data, using geometrical binning and statistical errors only, as the systematic errors are dominated by hadron decay uncertainties and largely correlated among different $p_{T}$-bins [10]. We find a minimal value of $\chi^{2} /$ d.o.f. of 1.2 for the combination $\mu_{R}=\mu_{D}=0.5 p_{T}$ and $\mu_{F}=2 p_{T}$ for the BFG I and II FFs and somewhat larger for GRV NLO, which is in good accordance with our observation above that $\mu_{D}=0.5 p_{T}$ should be preferred. Although QCD corrections beyond NLO are of course in principle important, they can be subsumed by an appropriate choice of scale. We have exploited this freedom by normalizing the theory to the data, in this way effectively fitting the higher-order terms. Note also that when $\mu_{D}$ falls below the starting scale $Q_{0}=\sqrt{2} \mathrm{GeV}$, numerical results from the BFG parameterizations of the FFs are no longer available 
and $\mu_{D}$ must at least be frozen there. In order to avoid the appearance of large logarithms (like $\log \mu_{R} / \mu_{D}$ ), we have chosen to freeze all three scales $\left(\mu_{R}, \mu_{F}\right.$ and $\left.\mu_{D}\right)$ at $Q_{0}$ in the short- and long-distance parts of our calculation. The error committed in this way is then at least of next-to-nextto-leading order, coming only from the uncompensated parts in the PDF and FF evolutions, and it affects all three FFs in a similar and only logarithmic way, ensuring a subdominant impact on our comparison with data. The goodness of our fit and its independence of the choice of FF can also be observed in the high- $p_{T}$ region of Fig. 3 (top).

We can then perform a $\chi^{2}$ test of the three different FFs in the signal region ( $p_{T}<5 \mathrm{GeV}$; see Fig. 3) (bottom), finding an acceptable minimal value of $\chi^{2} /$ d.o.f. of 2.8 for BFG II, while the BFG I and GRV NLO hypotheses lead to significantly larger values of 5.2 and 4.5 , respectively, and can be rejected at a confidence level of $99 \%$. Looking at Fig. 3 (bottom), these values of $\chi^{2} /$ d.o.f. are obviously dominated by the exceptionally high point at $p_{T}=4.25 \mathrm{GeV}$, which together with the point at $p_{T}=4.75 \mathrm{GeV}$ comes from the real-photon analysis. Although the other data points from the nearly real-photon analysis overlap with these two real-photon data points within their respective $p_{T}$-correlated systematic errors (see Fig. 2 of Reference [10]), the systematic errors differ among the two analyses. If we omit the two real-photon data points from the fit, we then find values of $\chi^{2} /$ d.o.f. of 0.68 for BFG II, 0.61 for BFG I and 0.63 for GRV. The current level of statistical (nearly real photons) and systematic (real photons) precision thus does not yet allow one to obtain stringent information on the photon FF. An improvement of about a factor of five in the statistical error would still be needed to apply our method successfully.

\section{Conclusions}

In this paper, we have seen that the combined virtual- and real-photon data from PHENIX seem to favor the BFG II parameterization with its relatively large gluon distribution over BFG I and GRV. This observation is, however, driven by an exceptionally high real-photon data point, which overlaps with the virtual photon data only within its large systematic error. The published virtual photon data from PHENIX alone do not yet allow for a conclusive distinction of the three available photon FFs and would require a reduction in their statistical error of at least a factor of five.

In the absence of new $e^{+} e^{-}$data, e.g. from a Linear Collider, our study shows nevertheless the potential of future inclusive photon measurements at BNL RHIC and CERN LHC to constrain the photon FFs with hadron collider data. In fact, much higher luminosities of 574 and $526 \mathrm{pb}^{-1}$ have already been recorded in 2013 by PHENIX and STAR, respectively, in $p p$ collisions at BNL RHIC and 5-10 $\mathrm{pb}^{-1}$
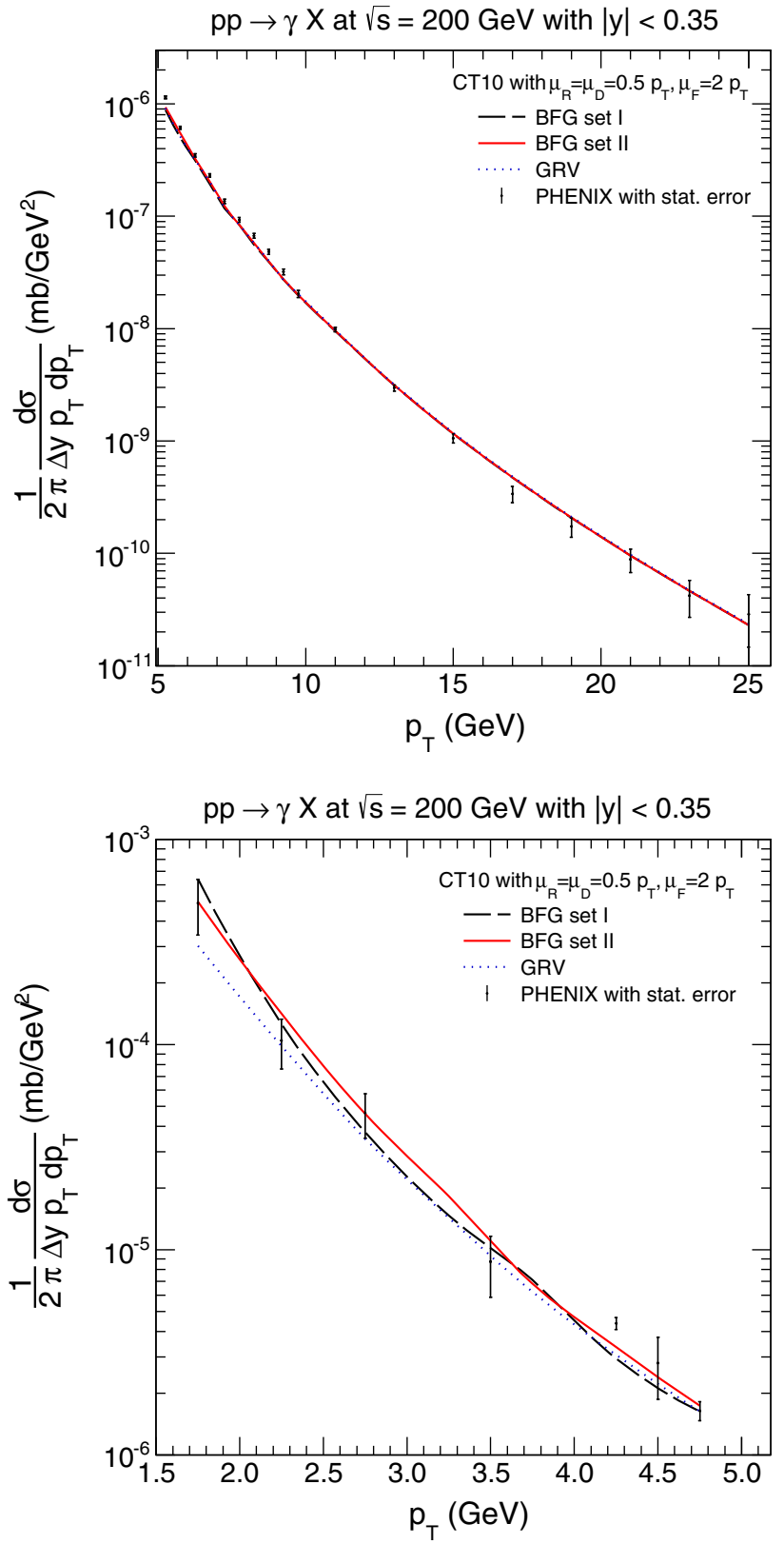

Fig. 3 Transverse-momentum distribution of inclusive photons as predicted by three different FFs and compared to PHENIX data with statistical errors only at high (top) and low $p_{T}$ (bottom) [10]

by the ALICE experiment at CERN LHC with $\sqrt{s}=7-$ $8 \mathrm{TeV}$. Unfortunately, at the LHC limitations of band width impede to trigger on low- $p_{T}$ data. For the suppression of meson decays, it seems crucial to exploit new experimental techniques such as electron triggers for nearly real-photon detection.

In the future it might be possible to also exploit photonjet correlations at BNL RHIC [21]. Indeed, photon-hadron correlations have already been studied, and the component of the photon momentum perpendicular to a trigger hadron has 
been extracted [22]. For decay and fragmentation photons, it was shown to be with about $0.5 \mathrm{GeV}$ significantly smaller than the one for directly produced photons $(\sim 0.8 \mathrm{GeV})$.

Acknowledgments We thank A. Gehrmann-De Ridder and Y. Yamaguchi for providing us with the FF routines and detailed information on the PHENIX data, respectively. We also thank C. Klein-Bösing and $\mathrm{J}$. Wessels for useful comments on the manuscript.

Open Access This article is distributed under the terms of the Creative Commons Attribution License which permits any use, distribution, and reproduction in any medium, provided the original author(s) and the source are credited.

Funded by $\mathrm{SCOAP}^{3}$ / License Version CC BY 4.0.

\section{References}

1. A. Adare et al., PHENIX collaboration. Phys. Rev. Lett. 104, 132301 (2010) arXiv:0804.4168 [nucl-ex]

2. M. Wilde, ALICE collaboration. Nucl. Phys. A 573c, 904-905 (2013) arXiv:1210.5958 [hep-ex]

3. M. Klasen, C. Klein-Bösing, F. König, J. P. Wessels, JHEP 1310, 119 (2013) arXiv:1307.7034

4. S. Abachi et al., HRS collaboration. Phys. Rev. D 40, 706 (1989)

5. D. Buskulic et al., ALEPH collaboration. Z. Phys. C 69, 379 (1996)

6. L. Bourhis, M. Fontannaz, J.P. Guillet, Eur. Phys. J. C 2, 529 (1998) hep-ph/9704447

7. M. Glück, E. Reya, A. Vogt, Phys. Rev. D 48, 116 (1993) (erratumibid. D 51, 1427, 1995)

8. H.L. Lai, M. Guzzi, J. Huston, Z. Li, P.M. Nadolsky, J. Pumplin, C.P. Yuan, Phys. Rev. D 82, 074024 (2010). arXiv:1007.2241 [hep$\mathrm{ph}]$
9. P. Aurenche, M. Fontannaz, J.-P. Guillet, E. Pilon, M. Werlen, Phys. Rev. D 73, 094007 (2006). hep-ph/0602133

10. A. Adare et al., PHENIX collaboration. Phys. Rev. C 87, 054907 (2013) arXiv:1208.1234 [nucl-ex] (Y. Yamaguchi, private communication)

11. T. Aaltonen et al., CDF collaboration. Phys. Rev. Lett. 111(4), 042003 (2013) arXiv:1303.6136 [hep-ex]

12. V.M. Abazov et al., D0 collaboration. Phys. Rev. D 88, 072008 (2013). arXiv:1308.2708 [hep-ex]

13. G. Aad et al., ATLAS collaboration. Phys. Rev. D 89, 052004 (2014). arXiv:1311.1440 [hep-ex]

14. S. Chatrchyan et al., CMS collaboration. JHEP 1406, 009 (2014). arXiv:1311.6141 [hep-ex]

15. K. Koller, T.F. Walsh, P.M. Zerwas, Z. Phys. C 2, 197 (1979)

16. E.G. Floratos, C. Kounnas, R. Lacaze, Nucl. Phys. B 192, 417 (1981)

17. W.A. Bardeen, A.J. Buras, D.W. Duke, T. Muta, Phys. Rev. D 18, 3998 (1978)

18. G. Altarelli, R.K. Ellis, G. Martinelli, S.-Y. Pi, Nucl. Phys. B 160, 301 (1979)

19. K. Ackerstaff et al., OPAL collaboration. Eur. Phys. J. C 2, 39 (1998). hep-ex/9708020

20. A. Gehrmann-De Ridder, E. W. N. Glover, Eur. Phys. J. C 7, 29 (1999). hep-ph/9806316

21. Z. Belghobsi, M. Fontannaz, J.-P.Guillet, G. Heinrich, E. Pilon, M. Werlen, Phys. Rev. D 79, 114024 (2009). arXiv:0903.4834 [hep$\mathrm{ph}]$

22. A. Hanks, PHENIX collaboration. Nucl. Phys. A 830, 455C (2009). arXiv:0907.4825 [nucl-ex] 